\title{
NGĀ KARAKIA TAWHITO KI TE PŪTAKE O TE RIRI
}

\section{H. McGarvey*}

\section{Kupu whakataki}

Koia ko te kaupapa o tēnei atikara e aropa ${ }^{-1}$ nui nei ki ngā karakia tūturu i takina i roto i ngā rā whakamaumahara o Te Riri Pākehā, arā, e mōhiotia ana ko Te Pūtake o te Riri, tae atu hoki ki ngā karakia tawhito i oti i roto i ngā tau maha ki ngā wānanga mau rākau, ki ngā momo tohi me ngā tikanga o te ao Māori. Ko te tapu o ngā karakia tūturu o tēnei ao, he rerekē ki te tapu o ngā karakia o te ao tūāuiruri whāioio o ō tātou tūpuna (Wānanga Karakia Māori, 2000). Waimarie kai te mau tonu i te tokoiti puta noa i Aotearoa ètahi o ngā karakia tawhito o tua whakarere, me te aha kai te kaha haere te tipu o te mate oha ki te ako i ngā karakia Māori. Engari, ko te ara aka whai i ngā karakia tūturu ehara i te mahi tamariki, ehara mō te katoa, kai tūpono ka tānoanoatia. Kua kite ahau i wētahi, kua poka noa ki te raweke i ngā taonga a koro mā, nāwai, ā, kai raro e putu ana.

Ki tētahi kōrero hoki, ki te hē te whāwhā i ngā karakia tūturu, he tangata tonu te utu i te mea he atua hae ngā atua Māori (Milroy \& Temara, P., kōrero ā-waha, 2004). Ko te nuinga o ngā tāngata e mōhio ana ki ngā karakia tūturu he mea i tohua e ngā pākeke, i tohia rānei e te tohunga. He kāwai heke, he kura huna kai roto i te toto. Nō reira he hapa kore te taki i ngā karakia tawhito, ko te tohunga taki i ngā karakia tūturu he tangata e mōhio ana ki ngā kōrero tuku iho, ki ngā whakapapa, ki ngā tikanga, ki te reo rangatira, ki te whēkite i ngā tūtohu o te wā. He mōhio ia ki ōna marae, he mōhio hoki ōna marae ki a ia. Ehara te whai i ngā karakia tūturu i te mahi tamariki, i te

1 He kupu tênei nā Tūhoe mō te uru rākau kotahi, te uru whakaaro kotahi, koia te matua o te kōrero, te huanui o te kaupapa. mahi mā te kūare, te kore mōhio, koinei te tapu o tua whakarere (Wānanga Karakia, 2000). Ko te reo taki i ngā karakia tūturu kia mārama, kia koi, kia mauritau (Tutua, kōrero ā-waha, 2005). E tautoko ana i ngā kōrero a Shane Te Ruki o ngā hapū o Ngāti Te Unu, o Ngāti Kahukura o te iwi o Ngāti Maniapoto, arā, ko te karakia Māori ehara i te karakia patipati, he whakahau kē i ngā atua o te pō, i ō tūpuna atua, te kāhui o ngā atua. Otirā, he māmā noa iho te whaihanga ingā karakia tūturu ki ngā kaupapa huhua o te wā, ahakoa he aha te kaupapa (Waka Huia, 2018). I pēnei hoki māua ko taku pāpara, a Te Makarini Temara, i whaihangatia e māua he karakia Māori hei ako atu ki ngā tauira o te wānanga.

Nō reira, hai whakarei ake i te iho matua o Te Rākau a Tāwhaki, ko te whakapapa o te orokohanga o te ao (Whakaahua 1).

\section{Te orokohanga o te karakia}

Ki te aromātaitia te whakapapa o te orokohanga o te ao, koia anō te orokohanga mai o ngā karakia tūturu, te ahunga mai o ngā mea katoa (Temara, T., 2012). Koinei te kōrero a wēnei pou kōrero o Tūhoe, a Te Makarini rāua ko Tamati Kruger mō te ahunga mai i a Io. Ka takina ake te ingoa o Io i roto i ngā karakia tawhito o Mātaatua, arā, Te Kawa Maiorooro kai raro iho nei (Wānanga Karakia Māori, 2000). Koia pū hoki ngā kōrero a Tūtakangahau o Maungapōhatu mō Io i hopukina mai e Te Peehi:

Ko Io he reo nō neherā tēnā. He atua nō mua noa atu. Nāna i whakaputa i ngā atua katoa. Koia te tìmatanga o ngā atua (Best, 1972, wh. 1026-27).

Nō reira, kai roto i ngā karakia tūturu tēnei mea te whakapapa e karakiatia ana, e tātaitia ana. Kai 


\begin{tabular}{|c|}
\hline Io-nui \\
\hline Io-roa \\
\hline I \\
\hline Io-matua \\
\hline Io-mātāne \\
\hline Io-hīranga \\
\hline $\begin{array}{c}\text { Io-taketake } \\
\mid\end{array}$ \\
\hline Io-whiwhia \\
\hline $\begin{array}{c}\text { Io-rawea } \\
\mid\end{array}$ \\
\hline Io-matangaro \\
\hline Io-matua-kore \\
\hline Te Pū \\
\hline $\begin{array}{c}\text { Te More } \\
\mid\end{array}$ \\
\hline $\begin{array}{c}\text { Te Weu } \\
\mid\end{array}$ \\
\hline Te Aka \\
\hline Te Rea \\
\hline $\begin{array}{c}\text { Te Wao } \\
\text { I }\end{array}$ \\
\hline Te Kū \\
\hline $\begin{array}{c}\text { Te Whē } \\
\text { I }\end{array}$ \\
\hline Te Kore \\
\hline Te Pō \\
\hline
\end{tabular}

WHAKAAHUA 1 Te orokohanga mai o te ao (Kruger, kōrero ā-waha, 2004)

tēnā iwi anō ōna tātai kōrero, kai tēnā iwi anō ōna tātai whakapapa. Ko te karakia e whakaatu pū ana i ngā atua, i ngā ariki kai roto i aua karakia rā, i tōna hāngaitanga. I roto i a mātou o Mātaatua ko wētahi kupu o ngā karakia nei paku te rerekē, whānui rānei te rerekē, rerekē ā-kupu, rerekē $\bar{a}$-manawataki, rerekē te wairua o tēnā tohunga, o tēnā tohunga, o tēnā whare, o tēnā whare, kāore i ōrite noa (Ngaropo, kōrero ā-waha, 2019). I te tau 2000, i tū tētahi wānanga karakia Māori ki
Te Teko, ko ngā tāngata i taua wānanga ko Te Hau Tutua, ko Te Pōroa Malcolm, ko Charlie Vercoe, ko Ben Mamaku, ko Eru Pōtaka-Dewes, ko Hirini Mead, ko Te Ururoa Flavell, ko Wetini Mìtai-Ngatai, ko Laurie Nicholls me te kaituhi kōrero ko Pakake Winiata. Ko te kōrero i takoto ki reira i ahu kē mai te karakia i Te Pō, i a Io. Ko tētahi kupu kī i whakahuatia i tērā wānanga ko te ihu tō mai i Te Pō (Wānanga Karakia, 2000). Koinei tētahi āhuatanga i tino tapu ai ngā karakia tūturu o te ao tawhito, nā te mea e whakataki ana te tohunga i ngā whakapapa tapu mai i a Io, mai i Te Pū, mai i Te Kore, mai i Te Pō, hekerangi mai ki ngā tamariki atua a Rangi rāua ko Papa. Koinā tētahi rerekētanga o ngā karakia Māori ki ngā karakia Karaitiana nei, ko te whakapapa. He karakia mō ngā wāhi katoa, mō ngā āhuatanga katoa o te ao nā te mea i ahu mai ngā mea katoa i te ira atua (Milroy \& Temara, P., kōrero ā-waha, 2004).

\section{Ko lo}

He maha ngā karakia tawhito e taki ana i te whakapapa o te orokohanga mai o te ao, e ai ki ngā tātai kōrero kua oti te whakatakoto i mua rā, e noho mai ana a Io i Mātangireia, ki tētahi whakahua ko Mātangi-ā-reia. Ko Mātangireia te whare kai Te Toi-o-ngā-rangi, kai te ngahuru mārua o ngā rangi tūhāhā, ā rānei ko Tikitiki-o-rangi. Ko te kōrero a te ruānuku o Ngāpuhi, a Māori Marsden, e noho ana a Io i Te Korekore (Marsden, 1975). Mēnā ki te rongo te taringa areare i wēnei rerenga ingoa ko Io tonu tēnā, ko te mauri, ko te kawa tiketike rānei i ahua mai i Tikitiki-o-rangi. He kawa tawhito tēnei nā te ruanuku, nā Te Hau Tutua o Ngāti Awa, o Mātaatua i whakaaro nui mai. Kai roto ko te whakapapa mai i a Io, tatū noa ki a Mātangireia, Tikitiki-o-rangi, ki Te Pū, heke mai ki Te Kore, Te Pō, ko Ranginui rāua ko Papatūānuku, ki te whai ao, ki te ao mārama:

\section{Te Kawa Maiorooro}

Ka takina te Kawa

Ko te Kawa-nui

Ko te Kawa-roa

Ko te Kawa-whakatiketike i ahua mai nō

Tikitiki-o-rangi

I tupu ko Te Pū

Ko Te Rito

Ko Te Weu

Ko Te Take

Ko te pūkenga

Ko te wānanga

Ko te taura

Ko te tauira 
Tēnei rā te awhi-nuku, te awhi-rangi

Nō te orooro o Io-matua-nui

I puea ia i te taketake i Ueue-nuku, i

Ueue-rangi

Tēnei ka hohou ki runga i te tipua

Ki runga i te tawhito

Ki runga i te kāhui o ngā Atua

Kia puta ēnei tauira ki te whai ao

Ki te ao mārama

Haumi e ... Ui e ... Taiki e!

(Tutua, kōrero ā-waha, 2007)

Ko ngā iho matua o te kawa maiorooro kai roto tonu i te reo, arā, he karakia tawhito tēnei hei whakatuwhera whare hou, he karakia whakatō, he whakaoho rānei i te mauri o te kāhui o ngā atua ki roto ki ngā pūkenga, ki ngā taura, ki ngā tauira kia mau puku i a rātou ngā kai mārō o te whare wānanga. Ko te orooro o Io-matua-nui kia tipu kaha ngā tātai kōrero taketake ki roto ki ngā taura me ngā tauira, kia noho ko rātou ā tōna wā hai tohunga mō te wānanga.

\section{Te ara o Tāwhaki ki ngā rangi tuehu}

E ai ki ngā kōrero tawhito o Mātaatua, i pikitia e te atua tangata nei, e Tāwhaki, te tuangahuru o ngā rangi tuehu ${ }^{2}$ ki te tiki i ngā taonga tapu a te tohunga ruānuku, a Tama-i-waho, ko te

2 He kupu nā Tūhoe mō ngā rangi tūhāhā. mātauranga me te karakia. E noho ana a Tamai-waho i te tuangahuru o ngā rangi, e kīia nei ko Tūwarea, ko Naherangi, ko Tawhitorangi. Kai roto i tētahi waiata tawhito e kōrero ana mō te ara o Tāwhaki. Arā:

Piki ake, kake ake

I te toi huarewa

I te toi ariki

I te toi urutapu

I te toi ururangi

I te toi ururoa

Te ara o Tāwhaki

I piki ake ai

Ki te Rangi o Ngā Atua e!

(Mead, 1996, wh. 61)

Anei te ara o Tāwhaki, koinei te ara tukutuku pūngāwerewere, te ara tōnakenake ki tua. Whāia ai e ngā tohunga o Mātaatua tēnei ara (Ripanga 1).

Rokohanga atu a Tāwhaki ki Naherangi ki a Tama-i-waho, ka akona a Tāwhaki e Tama-iwaho ki ngā karakia tapu. Inā tā Mead:

Ka tākina e te koroua rā ngā karakia huhua. Ko Te Whatu tēnā, ka whakamaua. Ko Te Āteateānuku tēnā, ko Te Āteateārangi tēnā, ko Hurihangate-pō, ko Te Mata, ko Te Korue-hī-nuku, ko Te Whēkite, ko Te Whakairihia, ko Te Huri, ko Ngā Puke, ko Te Tao-iti-ā-Paekohu, ko Ngā Mauri,

\section{RIPANGA 1}

\section{TE ARA O TĀWHAKI}

\begin{tabular}{|c|c|c|}
\hline Tuangahuru & $\begin{array}{l}\text { TŪWAREA, NAHERANGI, TAWHITORANGI } \\
\text { Ko Rehua te atua }\end{array}$ & \multirow{4}{*}{$\begin{array}{l}\text { Kāinga o ngā atua } \\
\text { Ngā Rangi a Rehua }\end{array}$} \\
\hline Tuaiwa & WAIRUA & \\
\hline Tuawaru & AUKUMEA & \\
\hline Tuawhitu & AUTOIA & \\
\hline Tuaono & $\begin{array}{l}\text { NGĀ ATUA } \\
\text { Ko Tāwhaki te atua }\end{array}$ & \multirow{3}{*}{ Ngā Rangi a Tāwhaki } \\
\hline Tuarima & $\begin{array}{l}\text { NGĀ TAUIRA } \\
\text { Kāinga o ngā atua iti }\end{array}$ & \\
\hline Tuawhā & $\begin{array}{l}\text { HAUORA } \\
\text { Kai konei Te Waiora-a-Tāne }\end{array}$ & \\
\hline Tuatoru & $\begin{array}{l}\text { NGĀ ROTO } \\
\text { Ko Maru te atua }\end{array}$ & \multirow{3}{*}{ Ngā Rangi a Maru } \\
\hline Tuarua & $\begin{array}{l}\text { WAKA MARU } \\
\text { Kāinga o te ua, o te rā }\end{array}$ & \\
\hline Tuatahi & $\begin{array}{l}\text { KIKO-RANGI } \\
\text { Ko Toimau te atua }\end{array}$ & \\
\hline
\end{tabular}

White, 1887, wh. 112 
ko Taku-ara-waerea, ko Tūtapa-ninihi, ko Ngā Wetewete. Nā, he tino maha ngā karakia i ākona e te koroua rā (Mead, 1996, wh. 66).

Ka whāia e ngā tohunga ahurewa o Mātaatua te ara o Tāwhaki i roto i ngā karakia tawhito pēnei i te kawa whakanukunuku:

Ka takina anō te kawa

Ko te kawa tuatahi

Ko te kawa tuarua

Ko te kawa tuatoru

Ko te kawa tuawhā

Ko te kawa tuarima

Ko te kawa tuaono

Ko te kawa tuawhitu

Ko te kawa tuawaru

Ko te kawa tuaiwa

Ko te kawa tuangahuru

Ka takina ko te kawa ...

Ka takina te kawa ki a Tū-mata-uenga

E Tū ... whakarongo mai rā ki te Ahurewa

I tupu iho ko te mauri areare

He mauri ngāhoa

He mauri taketake

I ahu mai nō Tikitiki-o-te-rangi

Tērā koa he Ahu-nuku, he Ahu-rangi

I takina mai i te whatinga o te tohi ahurewa $\mathrm{i}$

Tumu-whakairia

He ito ka tāpoua

He ito ka whakamaranga iho

Tēnā maranga iho kia eke

Haumi e... Ui e ... Taiki e!

\section{Ka ohu ngā uri o Tāwhaki}

Ko te tuangahuru o ngā rangi tuehu ka takina e ngā tohunga o Mātaatua ki te rā whakapōtaetanga o Te Whare Wānanga o Awanuiārangi i tū i ngā tau 2017-2019. Ka ohu ngā tohunga o Mātaatua ki Te Mānuka Tūtahi, ka ohu ngā uri o Tāwhakinui-ā-rangi, o Tāwhaki-nui-a-Hema. Koia tēnei karakia tūturu Māori e whai nei i te ara tukutuku o poutama ${ }^{3}$ ki te mātauranga mutunga kore, whenua noa. E whakatātūtū nei i ngā poupou o te whare kōrero o Awanuiārangi, ko Awanuiārangi he tipuna heke iho mai i a Tāwhaki. Koia tēnei:

Uia rā! Uia rā!

Uia rā ngā poupou o te whare kōrero! I poua mai koe i hea?

I poua mai koe i Mātangi-ā-reia, i Te Toi o ngā Rangi, ko te whare tēnā o Io Matua

Kore . . .

3 Te ara o Tāwhaki i pikitia e ia ki ngā rangi tuehu.
Ko Io-nui, ko Io-roa, ko Io-hīrangaranga, ko Io-te-pūkenga, ko Io-te-wānanga!

Rokohanga mai e Tāne-nui-ā-rangi ko Ngā Kete o Te Wānanga

Tūāuri, tūātea, tūāaronui!

Ka turuturua, ka poupoua kia puta te ira tangata ki te whai ao, ki te ao mārama e!

I poua mai koe i hea?

I poua mai koe i tō tīpuna ko Tāwhaki-nui-o-te-rangi

I pikitia e Tāwhaki te aka matua ki tua, rokohanga mai ko Tama-i-waho

Ko ngā whatukura mauri o te wānanga

Whēkite kia kite

Whēkaro kia rongo

Werohia kia mōhio

Te Whakairihia kia mārama

Kia mau te aka matua, kai mau ki te aka tāepa e!

I poua mai koe i hea?

I poua mai koe i te Hawaiki-nui, Hawaikiroa, Hawaiki-areare

I hoea mai Te Moana-nui-a-Kiwa e ō tūpuna ki Hawaiki-tautau! Ki Aotearoa nei e!

I poua mai koe i hea?

I poua mai koe i ngā whare wānanga, i ngā whare maire, i ngā whare kaha, i ngā whare kōrero o tua whakarere

Te mana, te tapu, te ihi! Tūpāpakurau e hā!

I poua mai koe $\mathrm{i}$ hea?

I poua mai koe i ō maunga, i ō awa, i ō kōhatu, i ō whenua, i ō taniwha, i ō iwi, i ō tūpuna ...

I puta ai te kōrero, "He tangata ākona ki te whare, tū ana ki te marae, tau ana ki te ao!"

I poua mai koe i hea?

I poua mai koe i te pūwāwātanga o Awanuiārangi wānanga rau .. .

He uri mākōkō nō ngā mātāwaka, nō ngā tai e whā ...

Ka whakatau ake ngā poupou o te whare kōrero i konei!

Tūruki, tūruki!

Paneke, paneke!

Tūruki, tūruki!

Paneke, paneke!

Haramai te toki!

Haumi e... hui e ... taiki e!

Ki te wetewetehia ngā kupu o te karakia o runga ake nei ka kitea ngā whaihanga o te hinengaro mākoi i hāngai ai ngā kupu ki te kaupapa o te hui. Anei anō tētahi karakia tūturu, nā taku pāpā, nā taku ruānuku, nā Te Makarini Temara i whakairo 
ngā kupu o te karakia nei. Ko tētahi pūmanawa whakahirahira o te ruānuku ahorangi ko tēnei mea te takutaku, ko te takutaku he whakahāngai i ngā karakia Māori ki te kaupapa e tū mai rā i mua i a koe, kāore e hapa. Ko Te Makarini tētahi i a ia tērā mana. Ko tēnei karakia he karakia whakatuwhera i tētahi waharoa hou. Kai roto i ngā kupu o te karakia nei ka kitea ngā atua kaitiaki ka whakahauhia e te tohunga ki runga i tēnei taonga a Tāne-whakapiripiri:

\section{Karakia Waharoa}

Tù mai ara

Tù mai waha

Tū mai te arataki nui

Te arataki roa

Tū mai te arataki matua e

Uru mai nunui, uru mai roroa, uru mai taketake

Te urunga tomo he tau..i..ira e..e..e

He urunga pūmoto nō ueuenuku, nō ueuerangi

He whakatau nōna māreikura, nōna whatukura

Ki konā koe e Tū, tū mai awa, tū mai ara, tū mai waha

Ko Tū-whakanguha, ko Tū-ka-riri, ko Tū-kaniwha, ko Tū-kai-tauā e

Ko te matamata ariki a Rongo ki konei

Ko Rongo-maraeroa, ko Rongo-mātāne, ko Rongo-mai-tahanui

Ko Rongo-whakaata, ko Rongo-taketake, ko Rongo-mau-tonu e

He rongomaiwhiti nō tawhito nuku, nō tawhito rangi

Hei whakanuku teretere kia hiwā

Koia ka whakairia, ka whakatārewa tū ki te rangi

Whano, whano, tū mai e ngā toki

Haumi e, ui e..e..e

Taiki..e..e..e

(Temara, T., kōrero ā-waha, 2016)

\section{Te ara o Tū}

Ka eke te rā o Te Riri Pākehā, ka moata te oho ake a ngā kaingārahu, ngā kaiwero me ngā kairākau. Kai te pōuri tonu te ao. Kātahi ka takahi atu ngā tāngata nei ki te wai tohi tauā, he wai tapu, he rerenga waiheke, kai te moana rānei, kai te awa rānei. Te taenga atu ki reira ka unuhia ngā kākahu, he kirikau anake, e mau ana ngā toa i ngā rākau matarua a Tū. Kātahi ka ārahina te ope kairākau nei e te ika ā-whiro, e te ruānuku rānei ki roto i te kōawa, i te roto moana rānei. I ētahi wā, kua oti kē te takoto o te rere o ngā karakia tohi nei, à rānei ka waihohia mā te wairua o ngā atua o te pō e tohu mai. Heoi, nō te kuhutanga atu ki roto i te wai ka takina ngā karakia tohi ka uwhiuwhihia te pahī tauā ki te wai. Ko ngā karakia he karakia whakapūmau, he whakaaraara, he whakaohooho kia tiu, kia oha, kia rea, kia toa te pūtauā. Kia pūmau te ngākau torokaha, te manawa whirikaha ki roto i a rātou ki te mura o te ahi.

Kua oti ngā kōrero mō te karaka whati, ko te rau o te karaka tēnā kai te ringa e tāuwhiuwhi ana, e ruirui ana i ngā toa ki te wai. Pērā anō te tohi, he tō mai i ngā atua o te pō kia tipua ai, kia whetē ai te ope kaiāwhā, kia ihonui, kia hiwa ki te pae o te riri. Ko te whakamutunga o ngā karakia tohi ko 'Tūturu whakamaua kia tinā. Hui e . . . Tāiki e!.' Oti ana te waitohi tauā, ko te tikanga tūturu kāore e kai, e ō ata rānei. Ko te karakia nei heria ai hei karakia tohi, hei tauparapara, hei karakia rānei i ngā toki tārei waka, toki whakahekeheke i ngā mahi toi whakairo. Nō Hawaiki-nui te karakia nei, i mauria mai i runga i te waka o Mātaatua. Koinei tā Tūhoe heri i te karakia nei, e mōhio ana ahau:

Toki uriuri, toki uri, toki amoamo

Aki hoki au i taku toki nei

Kia rahirahi mākiki, kia rahirahi mākaka

Nō hea te toki nei a manihi

Nō hea te toki nei a manaha

Te manaha nui a Tāne

Ka whakairia nui ake, nui ake, nui marire

Koia rā, koia rā

Koia rā Tū-tara-wiwini

Koia rā Tū-tara-wawana

Nō hea te toki nei

Nō runga i toki ai ōì

Hōmai nei taku toki

Taku toki nei he rīponga, he āwhenga

Tū te toki!

Haumi e

Hui e

Taiki e!

\section{Te ara o Rongo}

Kia tutuki te pōhiri me ngā wero, ngā mahi tū tauā, kātahi anō ka haere ngā ope tauā katoa ki muri, ki tētahi wāhi e taea ai e rātou te whakawātea i a rātou. Koirā te rerekētanga o te tohi mō te tokoiti, tērā ko te hohou rongo mō te tokomaha. Ka mātahi, arā, ka tū porowhita Te Akaaka Rauwhero, ka haere ngā poroaki, ngā mihi whakamutunga ki te ahi kā roa. Kātahi ka heria ngā karakia whakamoe i ngā rākau a Tū, whakairi i te patu, ā, ka whakahohou i te rongo ki waenganui i te katoa. Hei ètahi hohou rongo kua takoto he 
taonga ki te hau kāinga, nā te hau kāinga rānei ki ngā manuhiri haere mai. Kātahi ka huri ngā ope tauā o waho ki te whakaratarata ki te hau kāinga. Otirā, ko te whakakapinga kua noho te katoa ki te hākari, ki te whakangahau, ki te whakatutuki i te kaupapa whakahirahira o te rā. Anei he tauira o te karakia hohou i te rongo:

Rongopū, rongomau ...
Rongopū, rongomau ...
Ko te rongo i a wai
Ko Rongo-i-te-ioio
Ko Rongo-i-te-ieie
Ko Rongo-i-te-iaia
Te whare pātahi e hui te rongo
He rongo taketake
He rongo hurumanu
He rongo ka mau
He rongo ka ea
He rongo ka whiti rā
He rongomaiwhiti
Whiti ki tua, whiti ki waho
Whiti ki te whai ao, ki te ao mārama
Mai e te tipua
Mai e te tawhito
Mai e tawhiwhi ki a Rongo

(McGarvey, 2011)

\section{Te tohi tū-ora}

E ai rā ki ngā tātai kōrero a ngā tīpuna, ka whānau mai he tamaiti tāne ka tohia ki a Tū, ki a Rongo rānei, ka heria e te tohunga ki te wai tapu. I reira i te wai whakaika ka takina e te tohunga ngā karakia tū-ora, arā, kia tū tika, kia pakari, kia koi, kia ihomau te oranga ā-tinana, à-hinengaro, $\bar{a}$-wairua o te tamaiti rā. Kātahi ka whakahokia te tamaiti rā ki tōna matua whaea, ki te whare kōhanga. E rima, e ono rā i reira kātahi ka heria anōtia te tamaiti ka tūāina e te tohunga, ka tāuwhia ki te wai tauā hei hiki i te tapu kai runga i a ia, hei whakatō hoki i te aumangea, i te manawa toa, i te ihumanea ki runga i taua tamaiti kia noho ia i raro i te maru o Tū, kia mau tika katoa i a ia ngā mahi a Tū-mata-uenga.

Anei ètahi tauira o tēnei momo karakia te tūā me te whakamārama kai raro iho. Te nuinga o ngā karakia i hōmaihia ki ahau, nāku i whakamārama.

1. Korikori tama ki tūā

2. Ka riri ki tūā

3. Mau huata ki tūā

4. Kia niwha tama ki tūā

5. Mau patu tama ki tūā

6. Mau taiaha tama ki tūā
Ngā whakamārama

1. He wā tōna ka tipu te tamaiti rā hai toa.

2. Ka toa tū pakanga a tama.

3. Ka mau ia i te rākau huata.

4. Kua niwhaniwha katoa, arā, ka rikarika katoa ki ngā mahi a te toa.

5. Ka mau ia i te patu, i ngā momo patu katoa.

6. Ka mau ia i te taiaha.

Ko Te Tūā o Tū tēnei, ka pā ki te tamāroa anake.

1. Kia hāpai patu koe

2. Kia mau patu koe

3. Kia karo patu koe

4. Kia mau toa koe

5. Kia tāngaengae koe

6. Kia whetē koe

7. Kia ngāwari koe

8. Kia whiwhia ki te pehu o Tū

9. Oi whiwhia, oi rawea

10. Ka puta ki te whai ao

11. Ki te ao mārama.

Ngā whakamārama

1. Kia koi ki te whiu, ki te āki i te patu.

2. Kia koi, kia tau ki te mau i te patu me ōna karakia, ōna kōrero, ōna mahi katoa.

3. Ko te karo, ko te kakaro rānei kia kore ai e whara, e taotū.

4. Kia toa ngā mahi katoa.

5. Ko te tāngaengae koirā te ohonga o te aumangea o te toa. Kia oho, kia tiu.

6. Kia whetē te kanohi, arā, kia mataara.

7. Ko te tikanga o tēnei rerenga nā, ahakoa ka noho te toa i roto i te mura o te ahi, he ngāwari, he māmā noa iho te noho i roto i tērā āhuatanga, ka taea pea e ia te tāroi i te riri.

8. Kua kōrerotia ngā kōrero mō te peruperu, arā, pēnei anō te pehu, ko te uenga tēnei kia tau, kia oho, kia matapopore te toa i ngā wā katoa o te riri.

9. Kia mau te mana o Tū mō āke, āke, haere ake.

10. Koinei te manako nui kia puta te ihu i roto i ngā tātāwhāinga o te riri, kia toa, kia māia.

11. Kia tau hoki te oranga tonutanga nui ki runga i te toa, ki runga i tana whānau, i tōna hapū, i tōna iwi. Kia tau te māramatanga mutunga kore ki a rātou katoa, haere ake.

Tēnei anō tētahi karakia ka whakamahia mō te tūā, mō te tohi hoki i ngā pia.

1. Tūā kai te whiwhia, tūā kai te rawea

2. Kia tuputupu nunui e koe 
3. Kia tuputupu roroa e koe

4. Kia hōkai e koe, kia niwha e koe

5. Kia wehi e koe, kia whetē e koe

6. Kia toa e koe, kia tupu nui e koe

7. Kia toa e koe, kia tere e koe

8. Kia horo ki te hopu ika i te ati

9. Tamarahi kei a koe te ika i te ati

10. Ka māmā ki uta, ka māmā ki tai

11. Ka māmā ki ngā tūpuna

12. Ka māmā ki ngā ruahine

13. Ka māmā ki ngā taketake

14. Ka māmā ki te hau e tū nei

15. Kia uaua, kia toa, kia māia

16. Kia whetē, kia hōkai, kia tîkoro

17. Kia whetē ki ngā koromatua

18. Oi whiwhia! Oi rawea!

19. Tupe hinga, tupe takoto, tupe ara

20. Ka tau te ruhi, ka tau te ngēngē

21. Te hāmehameha o Tū

22. E Tū, whakaarahia!

23. Whano, whano, haramai te toki

24. Haumi e

25. Hui e

26. Tāiki e

\section{Ngā whakamārama}

1. Ko te tikanga o te tūā i konei he hiki i te tapu kai runga i te tamaiti, i te pia, oti rā he whakatau i te mana hāmehameha o Tū ki runga i te tamaiti rā.

2. Kia tipu ia hai tangata.

3. Kia tipu ia hai toa.

4. Kia rite ki te manu e hōkai ana i te rangi, e tiu ana i te wao nui. Kia niwhaniwha ki te pakanga.

5. Ka wehi, ka mataku te tangata ki a koe i runga i te whakapī me te whetē o tō kanohi me te mau i tō rākau.

6. Ka rongonuihia koe mō ngā mahi toa kai pakanga.

7. Kāore he tangata tere ake i a koe.

8. Māmā noa iho ki a koe te hopu i te ika i te ati, arā, te hoariri tuatahi ka patua i te māra o te riri. He tohu pai tēnei i roto i te pakanga.

9. E, he tino tangata koe, nāu i hopu te mata ika, te mata ngohi, te ika i te ati.

10. Kāore koe e wehi, e māharahara rānei mēnā he tū pakanga ki uta, ki tai rānei.

11. Kāore koe e wehi, e mānukanuka rānei ki ngā mākutu o te hoariri.

12. Kai a koe te taha ki tamatāne me te taha ki tamawahine, arā, ka taea e koe te raka i te matau, te raka hoki i te maūi (ruahine).

13. Kāore hoki koe e māwherangi ki ngā taketake, arā, ki ngā mōreareatanga, ki te hoariri me āna mahi huna, mahi whakamōkihi.

14. He tangata wehi kore ki te 'hau e tū nei', arā ko te hau mākutu tēnei, arā ko ngā mahi whaiwhaiā.

15. He uaua parāoa, he toa taumata rau, he māia whakaihurangi.

16. Ko koe pea te whakakanohitanga o te hōkioi manu taniwha. He toa horopū rākau.

17. Kāre e pāwerawera, e anipā rānei i waenganui i ngā toa rangatira o tōna kāinga, o te taha hoariri rānei.

18. Kia ù, kia mau, kia whita.

19. Ko te tupe he karakia tēnei hei whakamoe i ngā rākau o te riri, arā kia tupe hinga, kia hinga te hoariri, kia tupe takoto, kia takoto iwi pea te hoariri, kia tupe ara, arā, kua ara te rau o te patu ki te hahau i te ūpoko o tama à-hara.

20. Kua tāmōmōo te kaha o te hoariri, engari anō a manu ngārahu kai te hīkaka tonu, kai te hiakai pakanga.

21. Koinei te mana whakaihiihi o Tū-mata-uenga. Kāre i tua atu.

22. Kua oho a Tū.

23. Kua whakairohia ki te whatumanawa.

24. Kia ù.

25. Kia mau.

26. Mō āke tonu atu.

\section{Te tārehutanga o te toa kairākau}

Arā atu anō ētahi atu āhuatanga e kōrerohia ana i tēnei karakia tūā, arā, kia manawa pōhatu te māia ahakoa ngā wheiwheia, ahakoa te tokomaha pea o te hoariri, ko ia pea, ko te toa te apahau, arā, te whakatinanatanga tonu o Tū-mata-uenga. E kī ana tētahi tohunga rongonui o te ao Māori, a Reg Wharekura, koinei te tārehutanga o te tangata ki tētahi mea, arā, kia kotahi rāua, kia kotahi te mahi a te toa me te atua, ka whakaohongia i roto i a ia. Koia ko te toa te apahau o Tū, arā, he whaka-te-Tù-mata-uenga $\mathrm{i}$ a ia anō. E mea ana a Te Tairangi, ${ }^{4} \mathrm{ka}$ taea anō hoki e te tangata te tārehu i a ia ki te rākau, ki te kōhatu, ahakoa he aha te mea o te taiao. Ki tāna ko te tārehu he whakatau i te whakapapa Māori heke mai i te ao o wharawhara, arā nō te wherereitanga ${ }^{5}$ mai o te ao. Koia ka taea e te tangata te whakaoho mai i ngā kura huna kai roto i ōna toto, i tōna whakapapa, i heke iho i te rangi, $\mathrm{i}$ tipu ake $\mathrm{i}$ te whenua (Waka Huia, 2014).

4 Ko te ingoa wairua o Reg Wharekura.

5 Te orokohanga, te tïmatanga, te pütake. 
Waihoki, koinei hoki te āhuatanga ki te kaiwero ka whaka-te-manu i a ia ka puta atu ana ki waho ki te wero, anō he tīrairaka pāreherehe, à rānei he tīeke waewae tîkapekape, he kāhu matanui tū hokahoka, he kūaka mārangaranga nanahu papa teretere rānei e pīkarikari ana, e whakamìreirei ana i te marae ātea a Tū. Koinei te tārehutanga, he tiki atu, he whakaohooho i ngā atua, i ngā tipua, i ngā manu, i ngā kīrehe mohoao o tō tipuna whenua, o tō papa kāinga, o tō taiao. Kai roto i te whakapapa, kai roto i ngā pepeha, kai te tupuna whare hoki e iri ana, e tuhi ana, e rapa ana. Koinā rā te aronga matua ia o te para whakawai, o te whare tū tauā rānei, he whakaohooho i ngā taonga whakahirahira o te whare tū tauā a Tū-mata-uenga.

\section{Ngā Tohunga Ihorei Ki Te Akaaka Rauwhero 2013-2018}

Hei whakakapi ake i Te Rākau a Tāwhaki, ko ngā tohunga ihorei nā rātou i taki ngā karakia waitohi tauā huri taiāwhio i ngā akaaka o Aotearoa ki ngā rā whakamaumahara i Ngā Pakanga Whenua o Aotearoa.

\section{Rangiriri 2013}

Ope Tauā: Te Rau Takitahi a Tūhoe.

Tohunga Ihorei: Haturini McGarvey (Tūhoe), Tamahore Rangi (Tūhoe)

Waitohi tauā: Te awa o Waikato ki Ahurei.

\section{Pukehinahina 2014}

Ope Tauā: Te Rau Takitahi a Tūhoe.

Tohunga Ihorei: Haturini McGarvey (Tūhoe), Tamahore Rangi (Tūhoe), Tūtāwhiorangi Temara (Tūhoe), Te Kahu Apiata (Tūhoe), Wi Kamaua Hiakita (Tūhoe), Te Whetu McCorkindale (Tūhoe), Maaka Te Moana (Tūhoe)

Waitohi tauā: Ko te moana o Tauranga ki Maungatapu.

Ōrākau-Paewai 2014

Ope Tauā: Te Rau Takitahi a Tūhoe me Te Kawa Ariki o Tirikawa.

Tohunga Ihorei: Haturini McGarvey (Tūhoe), Awanuiārangi Black (Ngāti Pūkenga, Ngāti Ranginui, Ngāi Te Rangihouhiri).

Waitohi tauā: Te awa o Pūniu.

\section{Te Tarata 2015}

Ope Tauā: Tohenihorau, Te Rākau Hūmarie, Te Whare Toa o Ira, Te Kawa Ariki o Tirikawa, Te Tawharau Toa o Mātaatua.

Tohunga Ihorei: Haturini McGarvey (Tūhoe), Tamiaho Herangi-Serancke
(Waikato-Tainui), Wi Kamaua Hiakita (Tūhoe).

Waitohi tauā: Te awa o Waioweka.

\section{Ruapekapeka 2016}

Ope Tauā: Kōhao Rau, Te Tawharau Toa o Mātaatua, Te Kawa Ariki o Tirikawa.

Tohunga Ihorei: Paraone Tai Tin (Ngāti Hine, Ngāpuhi), Awanuiārangi Black (Ngāti Pūkenga, Ngāti Ranginui, Ngāi Te Rangihouhiri), Haturini McGarvey (Tūhoe).

Waitohi tauā: Te awa o Mōtatau.

\section{Maungapōhatu 2017}

Ope Tauā: Ngā uri o Tamakaimoana Tohunga Ihorei: Haturini McGarvey (Tūhoe). Waitohi tauā: Te awa o Waikare.

Te Kupenga a Taramainuku 2017 Ope Tauā: Kōhao Rau, Te Whare Toa o Ira, Te Tawharau Toa o Mātaatua, Te Kawa Ariki o Tirikawa, Te Akaaka Rauwhero.

Tohunga Ihorei: Akeni Tai Tin (Ngāti Hine, Ngāpuhi), Josh Te Kani (Ngāti Ranginui, Ngāi Te Rangihouhiri), Haturini McGarvey (Tūhoe), Manaakitanga Pryor (Ngāti Awa, Tūhoe) Wi Kamaua Hiakita (Tūhoe) Mutunga Rameka (Ngāpuhi), Muritere Isaiah (Ngāpuhi).

Waitohi tauā: Te awa o Rangitaiki-nui-ā-Tia.

\section{Kororāreka 2018}

Ope Tauā: Kōhao Rau, Te Tawharau Toa o Mātaatua, Te Kawa Ariki o Tirikawa, Te Akaaka Rauwhero.

Tohunga Ihorei: Akeni Tai Tin (Ngāti Hine, Ngāpuhi), Josh Te Kani (Ngāti Ranginui, Ngāi Te Rangihouhiri), Haturini McGarvey (Tūhoe), Manaakitanga Pryor (Ngāti Awa, Tūhoe), Wi Kamaua Hiakita (Tūhoe), Mutunga Rameka (Ngāpuhi), Muritere Isaiah (Ngāpuhi), Mori Rapana (Ngāpuhi). Waitohi tauā: Ko Te Rere-i-Tīria te awa.

\section{Kupu whakakapi}

Nō reira, kua takoto ēnei tātai kōrero mō ngā momo karakia tūturu i takina mai i te ao tawhito ki te ao hou, mai i ngā pakanga o mua ki ngā tau o Te Pūtake o Te Riri. I tēnei tau kai runga o Waitara, kai te take o Taranaki Te Pūtake o Te Riri. Ko te kaupapa o tēnei tau mō Te Pūtake o Te Riri ki Taranaki, ko 'Te riri me te raukura' (Wano, kōrero ā-waha, 2004). E whakatikatika ana ngā tohunga ihorei o tēnā pūtauā, o tēnā whare toa o 
te motu he aha ngā karakia tohi kura ki reira kia tutuki ai te taha ki a Tūmatauenga (te riri) me te taha ki a Rongo (raukura). Otirā, ka whakairia ake te kete awhekura o te wānanga ki konei.

Pōkai takarei te whare!

\section{Kāhui pukapuka}

Best, E. (1972). Tühoe: The Children of The Mist. A.H. \& A.W. Reed, Wellington, whārangi 1026-1027.

Marsden, M. (1975). God, Man and Universe: A Māori View. Kai roto i a King, M. (Eds.). Te Ao Huriburi: The Māori World Moves On. Hicks Smith \& Sons/ Methuen N.Z. Ltd, Auckland. Whārangi 157.

Mead, H. (1996). Tāwhaki Nui ā-Hema: Āna Mahi Whakahirahira. Reed Consumer Books. Auckland. Whārangi 58-67.
Temara, T. (2012). Te Haka Taparahi a Tühoe. Te Whare Wānanga o Awanuiārangi, Whakatāne. Whārangi 110-111.

Wānanga Karakia Māori. (2000). Uiraroa Marae, Te Teko.

White, J. (1887). The Ancient History of the Māori: His Mythology and Traditions. Vol.1. Government Printer, Wellington. Whārangi 112.

\section{Kiriata}

Temara, P. (2014, Mei 17). Waka Huia: Ōrākau. (Kiriata). Nō te wāhitau https://www.youtube. $\mathrm{com} /$ watch? $\mathrm{v}=\mathrm{C} 768 \mathrm{fGyuPnc}$.

Waka Huia. (2018). Shane Te Ruki. Nō te wāhitau https:// www.youtube.com/watch?v=fNXwHEZ4xjM. 